Methods the following data about endoscopists in screening programs between 201-017 were collected: age, sex, years as physician, specialization, annual colonoscopy volume. During 201-014, younger endoscopists $(<40$ yr $)$ underwent four meetings with a senior endoscopist (Cristopher Williams), to improve general endoscopic ability and, in particular, the sensibility to detect adenomatous lesions. ADR was calculated for each operator. Data were analyzed with SPSS program.

Results During the three year period, 191 endoscopists performed a total of 42,706 first colonoscopies after FIT positivity. One hundred forty with at least 20 colonoscopies after FIT positivity/yr, (mean age 48.2yr $\pm 10.8 \mathrm{yr}(\mathrm{M} \pm \mathrm{SD})$, Male $53.9 \%)$ were selected. Three hundred eight-two ADRs were collected during the observation period. Overall ADR was $43.7 \pm 9.5 \%$. Ninety-six of the endoscopists were specialized in gastroenterology, 35 in general surgery, and 8 in other special-

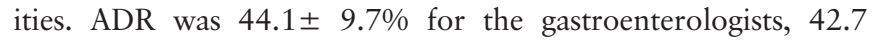
$\pm 9.6 \%$ for the surgeons $(p=n s)$ and $39.5 \pm 7 \%$ for those with other specialities $(p=0.033)$. ADR was significantly higher for the younger endoscopists (3-9yr 47.5\%; 4-9yr 44.6\%; $>50 \mathrm{yr} 41.4 \%$, with $\mathrm{p}<0.001$ ). The ADRs over the three year period were similar ( $44.2 \%$ vs $42.9 \%$ vs $43.3 \%, p=n s)$. No significant association between ADR and number of screening colonoscopies performed weew found. When the ADR in 2017 of 81 endoscopists was analyzed depending on the cumulative number of total colonoscopies/yr, it was found that the those with $>300$ colonoscopies/yr had higher ADRs ( $35.5 \pm 21.4 \%$ vs $43.5 \pm 11.5 \%, p=0.04)$ in colonoscopies after FIT positivity/yr .

Conclusions Study findings demonstrated that junior endoscopists and those specialized in gastroenterology were more effective in identifying adenomas during screening colonscopy procedures. High annual colonoscopy volumes and working exclusively as endoscopists but not years of experience or being older were associated with higher ADRs. Educational interventions seemed to be effective in improving sensitivity and performance.

\section{OWE-35 MULTIPROFESSSIONAL IN SITU SIMULATION IS AN EFFECTIVE METHOD OF IDENTIFYING LATENT PATIENT SAFETY THREATS}

Wisam Jafar*, Ms Debbie Suggitt, Elizabeth Uttley, Louisa Jackson, David Baxter. Stockport NHS Foundation Trust, Stockport, UK

\subsection{6/gutjnl-2019-BSGAbstracts.470}

Introduction Identifying errors in the ward environment, healthcare organisation or interaction between the multiprofessional team members is crucial. Such identification may prevent serious mistakes that can occur in high pressure gastroenterology emergencies. Unannounced multiprofessional in situ simulation can be an effective means of identifying latent patient safety threats before they occur. We set up a standardised in situ simulation programme centred around speciality specific emergency scenarios not only to improve skills, team working and communication but also to identify and address latent errors. The in situ faculty consisted of a consultant gastroenterologist; skills tutor; education fellow and director of medical education.

Methods We identified key learning objectives for several different emergency scenarios including: massive upper gastrointestinal haemorrhage; septic shock; post-procedure complications and cardiac arrest. Using a wireless high fidelity simulation manikin we ran 15 unannounced sessions between February 2017 and January 2019. There was a minimum of three faculty in attendance in each of the sessions and an overall total of 67 participants representing the spectrum of different professions including: doctors; nurses; health care assistants and students from nursing, medical, and physician associate backgrounds. Following completion of the scenario a structured debrief was conducted. Latent errors affecting patient safety at an individual, team or organisational level were identified and discussed. Following the scenarios, actions were put in place designed to address the identified errors in a timely fashion.

Results During the 15 unannounced sessions between February 2017 and January 2019 we identified the following latent errors: shortage of Sengstaken-Blakemore tubes, Bair Hugger and a step for CPR on the ward; lack of awareness as to how to activate the major haemorrhage protocol; junior doctors without working pagers; deficiencies in resuscitation skills amongst non-training grade doctors; members of the multiprofessional team forgetting each others' names and parts of the ALS algorithm; not knowing where the oral glucose gel is kept on the ward. Since the identification of these errors corrective measures have included: laminated ALS algorithms attached to the resuscitation trolleys and dedicated major haemorrhage protocol training at junior doctor induction. Equipment shortages including sourcing the relevant equipment has been undertaken by ward management. Dedicated training days for the non-training grade doctors has also been undertaken.

Conclusions In situ simulation is highly effective in identifying latent errors that could have potentially deleterious consequences on patient safety. This allows corrective measures to be undertaken in advance of real-life emergencies.

\section{Posters}

\section{PWE-100 ENDOSCOPY TRAINING IN SIERRA LEONE - SUCCESSFUL REMOTE SUPERVISION LEADING TO A SUSTAINABLE OGD SERVICE}

${ }^{1}$ Christopher Wells, ${ }^{2}$ Christopher Mountford, ${ }^{1} J o h n$ Hancock, ${ }^{1}$ Roisin Bevan*, ${ }^{3}$ Sorie Conteh, ${ }^{3}$ Donna Ese Thomas-Macauley, ${ }^{3}$ Finda Ngongo, ${ }^{2}$ David Nylander. ${ }^{1}$ North Tees and Hartlepool NHSFT, Stockton, UK; ${ }^{2}$ Newcastle Upon Tyne Hospitals NHSFT, Newcastle, UK; ${ }^{3}$ Choithram Memorial Hospital, Freetown, Sierra Leone

\subsection{6/gutjnl-2019-BSGAbstracts.471}

Introduction Sierra Leone is a poor country in Africa; population 7 million. Medical services are rudimentary. Until 2016 there was no established endoscopy service within the country. We describe a successful novel model of direct and remote supervision to train and mentor 3 doctors to competence in diagnostic Gastroscopy (OGD).

Methods A team of UK doctors developed and delivered a modified JAG OGD basic skills course during a week-long visit to Sierra Leone in 2016 to 3 doctors at Choithram Memorial Hospital, Freetown. Training involved group discussion of pre and post procedural aspects of OGD, intense procedural visual conceptualisation, hands-on model training and OGD on 12 volunteer patients under direct supervision. Trainees were trained to give feedback using the JAG DOPS form. Over the subsequent 12 months, the UK team offered regular 